\title{
Efectos de un programa de entrenamiento de la fuerza muscular sobre la condición física en adultos sexagenarios
}

\section{Effects of a Training Program on Muscular Strength on Physical Condition in Sexuagenarians}

\author{
L. B. Mendieta Toledo, ${ }^{1}$ L. R. Mendieta Toledo, ${ }^{2}$ J. M. Chamba Zambrano ${ }^{3}$ \\ http://dx.doi.org/10.21503/CienciayDesarrollo.2015.v18i1.03
}

\section{RESUMEN}

Objetivo: conocer los efectos de un programa de fuerza muscular en la condición física en adultos sexagenarios de Santo Domingo de los Tsáchilas-Ecuador. Materiales y método: se evaluaron a 44 participantes, distribuidos de manera aleatoria en dos grupos (ensayo controlado aleatorizado ECA), 0 (grupo control) $(n=22)$ con actividades de la vida diaria y 1 (grupo entreno) $(n=22)$ con entrenamiento basado en la fuerza muscular. Se evaluó antes y después de la intervención con pruebas físicas para determinar cómo valoración principal la condición física con el test de Cooper para la velocidad de la marcha y a través del índice esfuerzo percibido (IEP) según la escala de Borg, y fuerza muscular, como variables secundarias se evaluaron la velocidad de la marcha, fuerza-resistencia, peso corporal, índice de masa corporal (IMC), frecuencia cardiaca en reposo (FCR) y frecuencia cardiaca máxima (FCM). Se solicitó un consentimiento informado por escrito de los participantes. Se realizó una revisión médica de los pacientes antes del programa y un monitoreo durante el mismo. Resultados: los resultados arrojados en las pruebas fueron para el grupo entreno: mejora de la condición física con la prueba del (IEP), fuerza muscular, fuerza-resistencia y velocidad de la marcha, se disminuyó el peso corporal y el IMC; disminuyó la frecuencia cardiaca en reposo (FCR) y la frecuencia cardiaca máxima (FCM) en el grupo entreno. En el grupo control no existieron cambios significativos. Conclusiones: las conclusiones que se obtuvieron de este ECA es que la condición física de los participantes de un programa de entrenamiento de la fuerza muscular mejora la condición física y demás variables estudiadas de manera significativa en el grupo entreno en relación al grupo control.

Palabras clave: condición fisica, fuerza muscular, adultos sexagenarios.

\section{ABSTRACT}

Objective: To know the effects of a muscle strength program on physical condition in sexagenarians of Santo Domingo of Tsachilas-Ecuador. Materials and method: 44 participants were evaluated, who were randomly distributed in two groups (randomized controlled trial RCT), 0 (control group) $(n=22)$ with daily living activities and 1 (training group) $(n=22)$ with training based on the muscle strength. It was assessed before and after of the intervention with physical tests to determine as primary valuation, the physical condition with the Cooper test for gait speed, and through rating of perceived exertion

1 Magíster en Actividad Física y Salud. Docente investigador de la Pontificia Universidad Católica del Ecuador, sede Santo Domingo.Email:lbmendietat@pucesd.edu.ec

2 Profesor investigador de la Universidad Técnica de Loja, Ecuador. E-mail: leninrkd@gmail.com

3 Profesor investigador de la Universidad Técnica de Machala, Ecuador. E-mail:jmchamba66@hotmail.com 
(RPE) according to the Borg scale, and muscle strength as secondary variables were evaluated gait speed, strength-resistance, and body mass index (BMI), resting heart rate (RHR) and maximum heart rate (MHR). Written informed consent from participants was required. A medical examination of patients before the program was conducted as well as monitoring during treatment. Results: The results obtained in the tests were for training group: improvement of physical condition with IEP test; muscular strength, strength-endurance and gait speed, body weight as well as BMI decreased; both resting heart rate (RHR), and maximum heart rate (MHR) decreased with the trained group. In the control group, there were no significant changes. Conclusions: The conclusions drawn from this RCT is that the physical condition of the participants in a training program of muscle strength, improves physical condition and other variables studied significantly in the trained group compared to the control group.

Keywords: physical condition, muscular strength, sexagenarians.

\section{INTRODUCCIÓN}

Los estudios científicos avalan las intervenciones en adultos sexagenarios con programas de entrenamiento de la fuerza muscular, la condición física es uno de los componentes más importantes de la salud para las personas en general y para los adultos mayores en particular, M. J. Castillo Garzón, (2007; 17 (1):2-8) cita "El ejercicio físico, practicado de manera apropiada, es quizás la mejor herramienta hoy disponible para fomentar la salud y el bienestar de la persona”. La condición física en las personas es el conjunto de cualidades o capacidades motrices que son susceptibles de ser modificadas para su mejoría, a través del ejercicio físico, el termino incluye solo a aquellas cualidades que permiten ser trabajadas bajo niveles de exigencia rigurosos y con la efectividad de un programa bien diseñado, siguiendo unos objetivos determinados, la mejora de la condición física, disminuye la fatiga muscular y busca en sí la máxima eficacia mecánica, disminuyendo el riesgo de lesiones por actividades de la vida diaria, esta definición quedó circunscrita a tres condiciones básicas a partir de la década de los cuarenta: resistencia cardiovascular, resistencia muscular y fuerza muscular.

Para poder establecer cuáles son los factores determinantes en la condición física de los adultos, se profundizó en el vector que genera la situación problémica del problema y en sus derivaciones. El sedentarismo es en la actualidad el más acuciante problema que tienen los países en el mundo entero, llegando a porcentajes del $60 \%$ de inactividad física, en los países desarrollados hasta el 50\% de los adultos mayores no realiza actividad física, según las Estadísticas Sanitarias Mundiales (2012), de la Organización Mundial de la Salud (OMS) señalan que las tres principales causas de muerte en el mundo son la cardiopatía coronaria (isquémica), las infecciones de las vías respiratorias inferiores (como la neumonía) y los accidentes cerebro-vasculares, producido en muchos casos por el sedentarismo.

Las estadísticas Sanitarias Mundiales (2012), de la Organización Mundial de la Salud (OMS) también indican que el sedentarismo produce el aumento del peso u obesidad y se debe a la falta de actividad física, desde 1980, la obesidad se ha más que doblado en todo el mundo. Un dato importante a tomar en cuenta según la misma fuente es que: la obesidad puede prevenirse.

Los programas de entrenamiento de la fuerza muscular disminuyen estos antecedentes descritos en los párrafos anteriores, en el estudio de Natalia Bustamante Ara, et al (2012) en el que se evidencia una clara mejoría en el estado de salud de los adultos que participan de un progra- 
ma de entrenamiento de la fuerza muscular en el estudio Activity in Geriatric acute Care (AGECAR): rationale, design and methods; como se puede contrastar, el ejercicio de fuerza, son beneficiosos para la mejora de la condición física de los participantes de este tipo de programas, todo este bagaje de estudios científicos avalan la intervención en los adultos mayores sexagenarios.

Los programas de entrenamiento de la fuerza muscular son beneficiosos para mejorar la condición física y la aplicación de la escala de Borg, para medir el IEP, pueden contrastar esta aseveración, en el libro de García, Gil, M. (2012, cap. 3, pág. 27). Manual de ejercicio físico para personas de edad avanzada, sobre la prescripción física del ejercicio para edad avanzada sanas, cita "para que la actividad sea efectiva y tenga resultados esperados debe de estar bien programada, ajustada a las necesidades de cada persona y ser realizada de una manera sistemática”. El estudio se realizó siguiendo las normas éticas de Helsinki modificadas por última vez en la $64^{\mathrm{a}}$ Asamblea General, Fortaleza, Brasil, octubre 2013. Se realizó una revisión médica a cada uno de los participantes y el médico monitoreó mientras duró el estudio. Se extendió un certificado de idoneidad para realizar actividad física a cada participante.

En los entrenamientos de la fuerza muscular, existen factores adherentes que van concomitantemente trabajándose con él de manera congruente. Un factor es la velocidad de la marcha. En el estudio de Lenin Mendieta Toledo, Rijkaard Mendieta Toledo, Jonh Marcelo Chamba (2014), "El caminar es sin lugar a dudas una de las actividades físicas (AF) naturales del ser humano". De igual forma existe el factor de la resistencia de la fuerza que es influenciado por los programas de entrenamiento de la fuerza muscular; así, en el estudio de Cadore E.L., Pinto R.S., Bottaro M., Izquierdo M. (2014), Strength and endurance training prescription in healthy and frail elderly, citan: “... la manera de contrarrestar la fragilidad asociada a la edad y envejecimiento, el entrenamiento de la fuerza y la resistencia parece ser una estrategia eficaz para mejorar la hipertrofia muscular, fuerza y potencia de salida, así como el rendimiento de resistencia”.

La disminución del peso corporal y el índice de masa corporal son evidentes en un programa de entrenamiento de la fuerza muscular; así en el estudio de Tiffany N. Frimel, Favid R. Sinacore, Dennis T. Villarreal (2009) citan: “...el ejercicio reduce la pérdida de peso y aumenta la fuerza muscular en los adultos mayores frágiles".

En cuanto al incremento de la fuerza muscular con un programa de entrenamiento en diferentes grupos etarios, se puede evidenciar que en los adultos mayores, el incremento se logra con solo doce semanas de entrenamiento. Así, Mendieta L., Lucia A., Duarte, A. (2011) demostraron que en ancianos nonagenarios se logra incremento en la fuerza muscular, en Madrid- España; en otro estudio similar pero con un grupo etario de jóvenes, Mendieta L., Yaguachi F., Toledo K. (2013) lograron establecer las ganancias en fuerza muscular de los participantes en la ciudad de LojaEcuador. Estos estudios avalan esta intervención en un grupo de sexagenarios de la ciudad de Santo Domingo de los Tsáchilas.

\section{OBJETIVOS}

Conocer los efectos de un programa de fuerza muscular en la condición física en adultos sexagenarios de Santo Domingo de los TsáchilasEcuador

\section{MATERIALES Y MÉTODOS}

Se evaluaron a 44 participantes, distribuidos de manera aleatoria en dos grupos mediante un ECA, 0 (grupo control) $(\mathrm{n}=22)$ con actividades de la vida diaria y 1 (grupo entreno) $(\mathrm{n}=22)$ con entrenamiento basado en la fuerza mus- 
cular. Se evaluó antes y después de la intervención con pruebas físicas para determinar como valoración principal la condición física y fuerza muscular. Como variables secundarias se evaluaron la velocidad de la marcha, fuerza - resistencia, peso corporal, índice de masa corporal (IMC), frecuencia cardiaca en reposo (FCR) y frecuencia cardiaca máxima (FCM). Se solicitó un consentimiento informado por escrito de los participantes. Se realizó una revisión médica de los pacientes antes del programa y un monitoreo durante el mismo.

Los materiales que se utilizaron para determinar la condición física de los participantes del estudio fueron: test de Cooper para la velocidad de la marcha y para la valoración del índice metabólico y corresponde a $3,5 \mathrm{ml} \mathrm{O} / 2 / \mathrm{kg} \mathrm{x} \mathrm{min} \mathrm{(METS),}$ se valoró la fuerza de las piernas y brazos con el press banc; para las pruebas de resistencia de la fuerza se utilizó el test del escalón $(15 \mathrm{~cm}$ de altura para los sexagenarios), y las dominadas pruebas de fuerza para el tren superior e inferior.

Para determinar la prescripción médica del ejercicio se realizaron pruebas de FCR y FCM, así como la medición de parámetros físicos (ficha bioantropométrica) para el peso y el IMC.

Con los resultados obtenidos en la evaluación preintervención se diseñó un programa de entrenamiento en función de los mismos, con el objetivo de determinar los efectos del programa de entrenamiento de la fuerza muscular sobre la condición física de los participantes.

Se trabajó un ensayo controlado aleatorio (ECA, ofic. 10-12-2014). El Comité Ético de Investigaciones de la Pontifica Universidad Católica del Ecuador, Sede Santo Domingo (Santo Domingo, Ecuador, Investigium 2014-02) aprobó el diseño, los protocolos y el procedimiento de consentimiento informado por escrito, todos los participantes proporcionaron un consentimien- to informado por escrito (anexo 1). Después de las medidas realizadas al inicio del estudio, los participantes fueron asignados al azar a los grupos de control o de intervención, como se explica más adelante. Los dos grupos fueron evaluados antes de empezar el programa y de su designación. El grupo de intervención siguió un programa de entrenamiento de la fuerza muscular de 12 semanas (más una semana de ambientación y una semana de preevaluación) que fue seguido por una semana de evaluación postprograma por los mismos investigadores que realizaron las medidas iniciales del estudio. El estudio se realizó entre diciembre de 2014 y marzo de 2015 , siguiendo las normas éticas de la declaración de Helsinki, modificadas por última vez en la $64^{\mathrm{a}}$ Asamblea General, Fortaleza, Brasil, octubre 2013.

\section{Participantes en el estudio y criterios de selección}

Los potenciales participantes incluyeron a 58 sexagenarios residentes en la comunidad de Santo Domingo de los Tsáchilas-Ecuador, seleccionados del centro ambulatorio de la seguridad social del Ecuador, en Santo Domingo (ver diagrama de flujo de los pacientes en la anexo 2). A todos los participantes se les realizó un reconocimiento médico completo y se extendió un certificado médico del profesional que realizó el diagnóstico (anexo 3). Los criterios de inclusión fueron los siguientes: 60 años de edad o más, no tener previsto trasladarse de la ciudad de durante el estudio, capacidad de caminar sin ayuda, capacidad de comunicarse y capacidad y voluntad de proporcionar un consentimiento informado. Los criterios de exclusión fueron los siguientes: enfermedad aguda o terminal, infarto de miocardio en los últimos 3 meses, incapacidad de caminar, padecer enfermedad cardiovascular inestable u otra afección médica, fractura en las extremidades superiores o inferiores en los últimos tres meses, demencia grave, falta de volun- 
tad para cumplir con los requisitos del estudio o para ser asignados al azar al grupo de control o de entrenamiento, enfermedad neuromuscular o consumir fármacos que afecten a la función neuromuscular. Se emitió un certificado médico de monitoreo durante el programa (anexo 4) y una prescripción médica del ejercicio (anexo 5)

\section{Aleatorización y bloqueo}

Se asignó al azar a los participantes al grupo de control o al de entrenamiento, con un diseño de bloqueo para sexo y capacidad velocidad de marcha basada en la escala del Test de Cooper, según una secuencia de aleatorización generada por el ordenador de la marca HP.

El grupo de investigación desconocía la codificación utilizada para asignar los participantes a los grupos ( 0 para los cuidados habituales y 1 para la intervención). El personal de evaluación desconocía la asignación al azar de los participantes. Se informó explícitamente a los participantes y se les recordó que no hablaran sobre su asignación al azar con el personal de evaluación. No fue posible ocultar la asignación de grupos al personal que participó en el entrenamiento.

Finalmente iniciaron el estudio 44 participantes, 22 para cada grupo (anexo 1, diagrama de flujo). Los grupos eran homogéneos inicialmente para los principales datos demográficos, médicos y funcionales ( $p>0,05$ para todas las comparaciones entre grupos).

\section{Grupo con cuidados habituales}

Durante el estudio, los participantes del grupo control (cuidados habituales) siguieron los consejos generales de los entrenadores de realizar actividades de la vida diaria y los posibles efectos de los ejercicios de movilidad. Realizaron movimientos pasivos y de poca actividad durante 4045 minutos al día, 5 días a la semana, consisten- tes en una serie de estiramientos suaves y rítmicos de las articulaciones individuales.

\section{Grupo de intervención}

Los participantes asignados al grupo de intervención siguieron un plan de 5 sesiones de entrenamiento semanales consecutivos durante 12 semanas ( 60 sesiones en total). Cada sesión tuvo una duración aproximada de 45-50 minutos. El programa de ejercicios se centró en el entrenamiento de la fuerza muscular, cada sesión comenzó y terminó, respectivamente, con periodos de calentamiento y enfriamiento de baja intensidad (aproximadamente 5-7 minutos cada uno), que consistieron principalmente en ejercicios de estiramiento con implicación de todos los grupos musculares. La parte central de la sesión consistió en entrenamiento para el fortalecimiento muscular con implicación de los grandes grupos musculares de las extremidades inferiores, es decir, ejercicio de fuerza de presión de las piernas realizado con máquinas de resistencia variable de fabricación artesanal. Los participantes realizaron entre 2 y 3 series de 8 a 10 repeticiones con periodos de descanso de 1 a 2 minutos entre ejercicios.

La carga se fue incrementando gradualmente a medida que mejoraba la fuerza de cada persona, es decir, desde el $70 \%$ de 1 repetición máxima (1RM) al inicio del programa hasta el $100 \%$ de 1RM al final del mismo. Los ejercicios de estiramiento de los músculos implicados en los ejercicios anteriores se realizaron durante los periodos de descanso. Se aconsejó a los participantes que realizaran todos los movimientos de forma lenta y controlada, y que no contuvieran la respiración durante los ejercicios.

\section{Retención y cumplimiento de los participantes}

Para reducir el abandono de los participantes y cumplir con el programa de entrenamiento, todas las sesiones incluyeron música y se realizaron 
en un local ventilado y bien iluminado (Gimnasio GYM FACTORY y cancha de uso múltiple). Especialistas cualificados en entrenamiento físico supervisaron cada sesión de entrenamiento y trabajaron con grupos de 2 a 3 personas para asegurar que los participantes realizaran los ejercicios correctamente. Para el entrenamiento cardiovascular, se trabajó en conjunto.

\section{Familiarización y evaluación de la fiabilidad}

Antes de comenzar el estudio los participantes realizaron un periodo de ambientación de una semana con las pruebas descritas más abajo, las cuales consistieron en sesiones de aproximadamente 45 minutos. Cada sesión estuvo precedida por un calentamiento y terminó con un enfriamiento que consistió en las mismas actividades y la misma duración que el periodo de entrenamiento. Cada sesión de ambientación consistió en 1 o 2 series de 6 a 8 repeticiones de los ejercicios. También se evaluó y reevaluó la fiabilidad de cada criterio de valoración. Se obtuvo un alto coeficiente de correlación intraclase ( $R$ consistentemente $>0,98 ; p<0,001$ ) entre las pruebas repetidas para todas las pruebas estudiadas.

\section{Variables principales:}

\section{Condición fisica "Test de Cooper"}

Esta prueba si bien es cierto se trabaja para determinar la velocidad de la marcha y la distancia recorrida, sirve también para determinar la condición física de los participantes mediante la obtención de los Met's. El aumento en la distancia recorrida es un factor que determina la condición física de quienes realizan esta prueba. Se aplicó en una cinta rodante con la presencia de dos monitores a cada lado de la rampa rodante (trotadora eléctrica), con el fin de prevenir caídas en los participantes. Esta prueba consistió en caminar o correr durante 12 minutos
Para determinar el índice de esfuerzo percibido según la escala de Borg, se utilizó la misma prueba (test de Cooper), en donde a través de una prueba subjetiva se preguntó a los participantes sobre la percepción del cansancio frente al esfuerzo. Tuvo como objetivo monitorizar la intensidad del ejercicio mediante un sistema de delimitación que tiene en cuenta las respuestas del organismo frente a una intensidad de esfuerzo concreta y además de contemplar el esfuerzo, la respiración y las respuestas emocionales al ejercicio, también se trabaja la frecuencia cardiaca máxima.

\section{Velocidad de la marcha (Test de Cooper)}

Los participantes realizaron la prueba de caminar lo más rápido que ellos pudieran aplicando el test de Cooper (aplicado para los adultos mayores). Esta prueba se realizó con la presencia de dos monitores a cada lado de la rampa rodante (trotadora eléctrica), con el fin de prevenir caídas en los participantes. Esta prueba consiste en caminar o correr durante 12 minutos.

\section{Fuerza de piernas}

Se evaluó la fuerza muscular dinámica de las extremidades inferiores mediante un protocolo normalizado de la prueba de fuerza, con fuerza de presión de las piernas sentado con un máximo de 6 o 7 repeticiones ( 6 o 7 RM). El valor de 1 RM se estimó utilizando la ecuación de Brzycki: $1 \mathrm{RM}=102,78-2,78 \times$ número de repeticiones. Las cargas iniciales fueron de entre 70 y el 100 $\%$ del peso corporal. Después de un breve periodo de descanso (2 minutos) se añadieron incrementos de $4-6 \mathrm{~kg}$ hasta que el participante fuera incapaz de levantar la carga más de 6 o 7 veces, lo cual en general ocurrió después de 5 ensayos. Todos los participantes pudieron levantar la carga inicial al menos una vez. Se les enseñó la forma adecuada de respirar para cada movimiento y levantar la carga. 

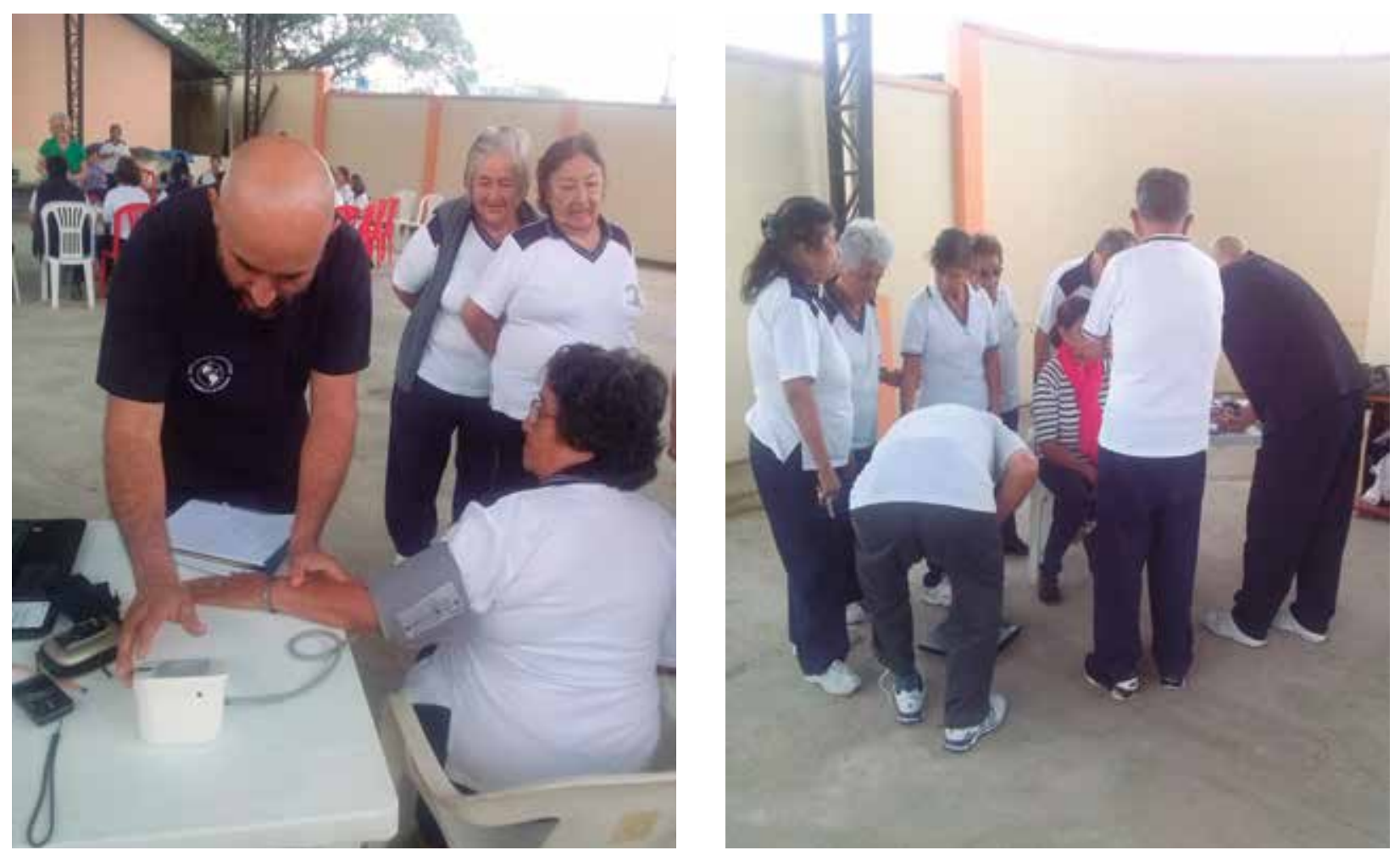

Figura 1. Adultos mayores de Santo Domingo de los Tsáchilas. Valoración del peso, índice de masa corporal, frecuencia cardiaca en reposo y frecuencia cardiaca máxima.

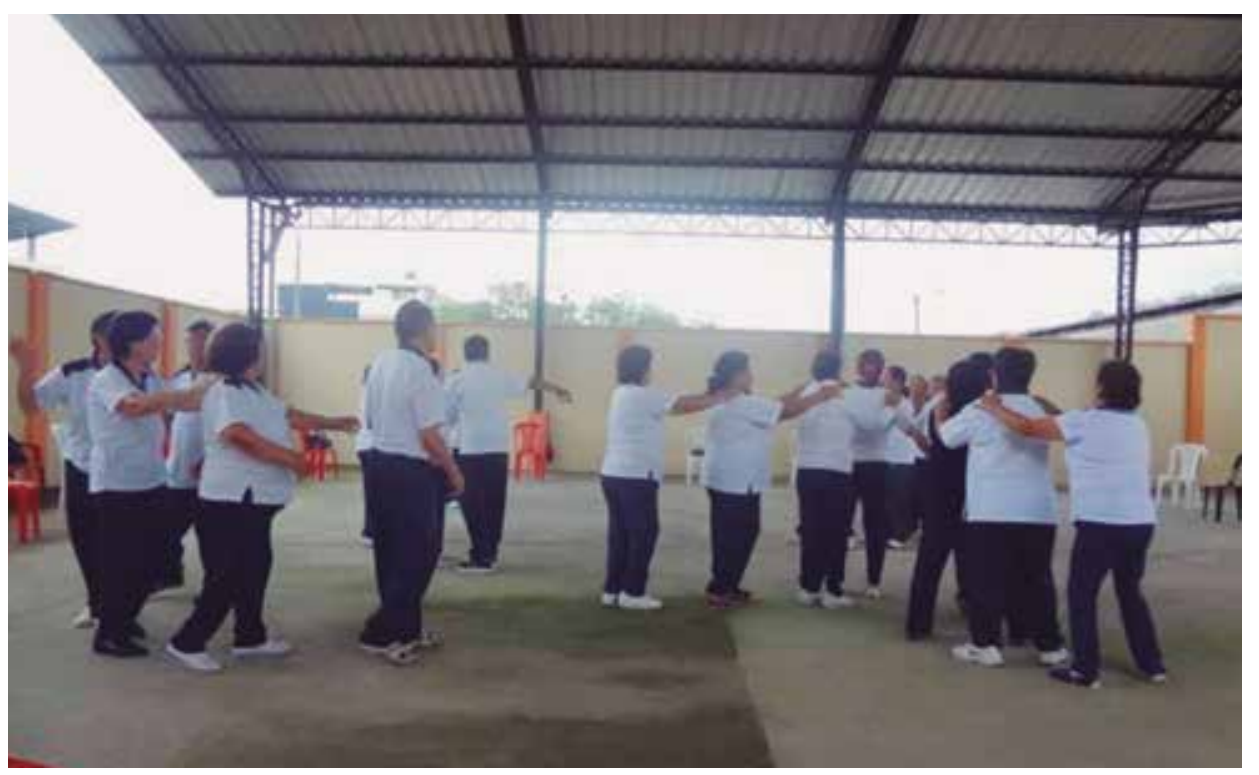

\section{Fuerza de brazos}

Se evaluó la fuerza muscular dinámica de las extremidades superiores mediante un protocolo normalizado de la prueba de fuerza, con fuerza de presión de los brazos acostado con un máximo de 6 o 7 repeticiones ( 6 o $7 \mathrm{RM}$ ). El valor de 1 RM se estimó utilizando la ecuación de
Brzycki: $1 \mathrm{RM}=102,78-2,78 \mathrm{x}$ número de repeticiones. Las cargas iniciales fueron de entre $70 \mathrm{y}$ el $100 \%$ del peso corporal. Después de un breve periodo de descanso ( 2 minutos) se añadieron incrementos de $2-4 \mathrm{~kg}$ hasta que el participante fuera incapaz de levantar la carga más de 6 o 7 veces, lo cual en general ocurrió después de 5 ensayos. Todos los participantes pudieron levantar 
la carga inicial al menos una vez. Se les enseñó la forma adecuada de respirar para cada movimiento y levantar la carga.

\section{Variables secundarias:}

\section{Resistencia de la fuerza de piernas}

Para determinar la condición física mediante la determinación de la resistencia de la fuerza de los participantes se realizó la prueba del escalón de Harvard, que determina la fuerza resistencia. Consistió en subir y bajar escalones de 15 centímetros de altura durante un tiempo determinado de cuatro minutos y contar las veces que realiza este ejercicio.

\section{Resistencia de la fuerza de brazos}

Para determinar la condición física de los participantes se realizó la prueba de las dominadas, que determina la fuerza resistencia. Consistió en flexionar y extender (subir y bajar) los brazos en una barra con el peso de su propio cuerpo y de manera continuada, con una altura determinada según el tamaño de los participantes, el aparato tenía regulación de su altura (cada 5 centímetros de altura)

\section{Peso e Índice de Masa Corporal (IMC)}

Se pesó con una báscula electrónica de la marca Sony, con un nivel de precisión de $0,5 \mathrm{~g}$, a todos los participantes en dos ocasiones (pre y post), para determinar las ganancias o pérdidas, y además se estableció el IMC en cada uno de ellos con la aplicación de un cálculo establecido por un software diseñado en la Universidad Europea de Madrid (UEM)

\section{Frecuencia Cardiaca en Reposo y Máxima}

La FCR se estableció enseñando a los participantes a medirse la misma en casa, durante siete días seguidos y al despertar, dejar cinco minutos antes de levantarse, tomarse la frecuencia cardiaca en el cuello, colocando los dedos índice y corazón en la zona indicada (pulso carotideo), presionando suavemente con los dedos y una vez se sienta las palpitaciones, contar cuántas pulsaciones tenemos en un minuto (o en medio minuto y multiplicarlo por dos). Anotar el número de pulsaciones, sumar las cantidades de los siete días y dividir para este número (7); el resultado es la FCR.

La FCM fue medida por cada uno de los participantes una vez finalizada la prueba de esfuerzo del test de Cooper. Consistió en tomar el pulso de la misma manera que en la FCR e informar al monitor para anotar el valor. Esta medición se la realizó antes de empezar el programa y al finalizar el mismo (pre y post). Para determinar la FCM que debía tener cada participante, la medición se sujetó a la formula FCM $=208,75-$ (0,73* edad), diseñada por Tanaka (2001).

\section{Análisis estadístico}

Para las comparaciones iniciales entre grupos (cuidados habituales frente a intervención) y para comparar la condición física y fuerza muscular, se analizaron las variables continuas con la prueba $\mathrm{T}$ de Student para datos no apareados (o su equivalente no paramétrico, la prueba $U$ de Mann Whitney) y pruebas de Chi cuadrado para datos nominales. Las comparaciones múltiples se ajustaron para evitar la significación masiva.

Para evaluar los efectos del entrenamiento sobre las variables del estudio de la condición física, velocidad de la marcha, IEP y fuerza del tren inferior y superior se analizaron los datos según el principio de intención de tratar. Cuando no se contó con datos posteriores a la prueba, se consideraron las puntuaciones obtenidas al inicio del estudio como puntuaciones de después de la prueba. Se utilizó un análisis de varianza (ANOVA) bifactorial (grupo y tiempo) con medidas repetidas. 
El análisis se repitió utilizando los valores iniciales como covariable. Para cada variable dependiente se informó del nivel de significación correspondiente a los efectos del grupo principal (entre sujetos), del tiempo (para cada sujeto) y de la interacción (grupo $\mathrm{x}$ tiempo). Con el fin de evitar un error tipo I se realizaron comparaciones post hot (comparaciones previas y posteriores en cada grupo, mediante la prueba de Bonferroni) solo cuando se obtuvo una interacción significativa. Para todos los criterios de valoración se realizaron pruebas de ANOVA para los 2 grupos enteros. El nivel de significación de la prueba se estableció en 0,05 .

\section{RESULTADOS}

\section{Cumplimiento y efectos secundarios}

La fidelidad del programa presentó hasta un $100 \%$ las evaluaciones postprograma, ya que todos los participantes culminaron el estudio. No se observó ningún efecto adverso ni problema de salud atribuible al entrenamiento indicado en ninguno de los participantes del grupo de intervención. Un participante padecía de dolor en la región lumbar transitoria al inicio del programa de entrenamiento y por tanto tuvo que reiniciar el mismo con una carga menor ( $50 \% 1 \mathrm{RM})$, lo cual le permitió terminar la intervención de manera satisfactoria.

\section{Resultados principales}

Se encontró efecto significativo del grupo, del tiempo o de la interacción $(\mathrm{p}=0,05)$ para la condición física. En la prueba del test de Cooper adaptado para adultos sexagenarios en protocolo en rampa rodante en la obtención de los Met's en el grupo control durante la prueba no se obtuvieron datos estadísticamente significativos, manteniendo el porcentaje en la pre y post evaluación; no así el grupo entreno tuvo ganancias en la postevaluación y bajaron los participantes que hacían $1200 \mathrm{~m}, 1500 \mathrm{~m}$, y subieron los que hacían 1800 m y 2200 m (Figura 1).

Tabla 1. Test de cooper (Met's)

\begin{tabular}{ll|cccc}
\multicolumn{6}{c}{ DISTANCIA RECORRIDA EN TIEMPO 12 MIN. } \\
\hline \multirow{2}{*}{ PRE } & METROS & $1200 \mathrm{~m}$ & $1500 \mathrm{~m}$ & $1800 \mathrm{~m}$ & $2200 \mathrm{~m}$ \\
& CONTROL (METS) & 2,81 & 3,79 & 7,21 & 9,29 \\
& ENTRENO (METS) & 2,81 & 3,79 & 7,21 & 9,29 \\
\multirow{2}{*}{ POST } & CONTROL (METS) & 2,81 & 3,79 & 7,21 & 9,29 \\
& ENTRENO (METS) & 1,81 & 1,79 & 9,21 & 10,29 \\
\hline
\end{tabular}

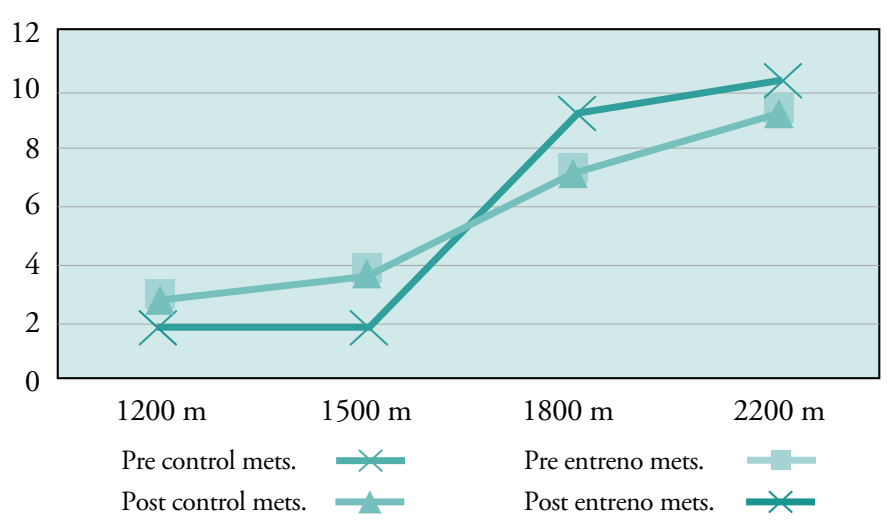

Figura 1. 
Se encontró efecto significativo del grupo, del tiempo o de la interacción $(\mathrm{p}=0,05)$ para la condición física, en la prueba del test de Cooper adaptado para adultos sexagenarios en protocolo en rampa rodante en el IEP de Borg; así, en el grupo control se produjeron datos estadísticamente significativos, más en el grupo entreno se pudo evidenciar que de cinco participante que consideraron muy dura la prueba en la preevaluación disminuyó a cuatro, de nueve que consideraron dura la prueba bajaron a seis, de cuatro que consideraron dura la prueba en el pre, bajaron a dos, y de cero que consideraron que era ligero y muy, muy ligero fueron cinco y tres los que así lo percibieron en el esfuerzo en la postevaluación (Figura 2).

\section{Tabla 2. Escala de Borg. (Índice de esfuerzo percibido)}

\begin{tabular}{|c|c|c|c|c|c|}
\hline & \multirow{2}{*}{ ESCALA } & \multicolumn{2}{|c|}{ G. ENTRENO } & \multicolumn{2}{|c|}{ G.CONTROL } \\
\hline & & PRE & POST & PRE & POST \\
\hline 0 & Reposo/Nada & 0 & 0 & 0 & 0 \\
\hline 1 & Muy, muy ligero & 0 & 3 & 0 & 0 \\
\hline 3 & Ligero & 0 & 5 & 0 & 0 \\
\hline 4 & Moderado & 4 & 2 & 4 & 2 \\
\hline 5 & Algo Duro & 0 & 2 & 0 & 10 \\
\hline 6 & Duro & 9 & 6 & 9 & 6 \\
\hline 8 & Muy Duro & 5 & 4 & 5 & 0 \\
\hline 10 & Extremadamente Duro & 4 & 0 & 4 & 4 \\
\hline
\end{tabular}

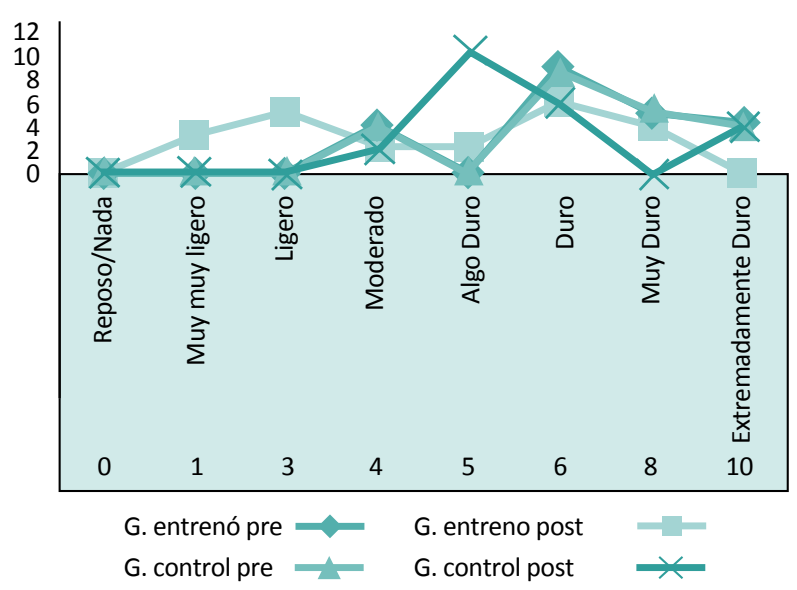

Figura 2.
Se encontró efecto significativo del grupo, del tiempo o de la interacción $(\mathrm{p}=0,05)$ para la velocidad de la marcha en el grupo entreno durante la prueba de test de Cooper se obtuvieron ganancias de un 9,09\% en el grupo que disminuyó de cuatro a dos participantes que hicieron 1200 metros; $0 \%$ en el grupo que se mantuvo con cinco personas que significó el 22,72 \% de los participantes que hicieron 1500 metros; ganancias del $4,5 \%$ en el grupo que aumentó de un 40,9 $\%$ a $45,45 \%$ en el grupo que aumentó de nueve a diez participantes que hicieron 1800 metros; $4,54 \%$ en el grupo que aumentó de un $18,18 \%$ a $22,72 \%$ en el grupo que aumentó de cuatro a cinco participantes que hicieron 2200 metros. (Figura 3).

Tabla 3. Test de cooper (velocidad de la marcha)

\begin{tabular}{|ccccc} 
VARIABLES & $\begin{array}{c}\text { Pobre } \\
1200 \mathrm{mts}\end{array}$ & $\begin{array}{c}\text { Regular } \\
1500 \mathrm{mts}\end{array}$ & $\begin{array}{c}\text { Buena } \\
1800 \mathrm{mts}\end{array}$ & $\begin{array}{c}\text { Muy Buena } \\
2200 \mathrm{mts}\end{array}$ \\
$\begin{array}{c}\text { CONTROL } \\
\text { POST }\end{array}$ & 5 & 8 & 6 & 3 \\
$\begin{array}{c}\text { CONTROL } \\
\text { PRE }\end{array}$ & 4 & 5 & 9 & 4 \\
\hline $\begin{array}{c}\text { ENTRENO } \\
\text { POST }\end{array}$ & 2 & 5 & 10 & 5 \\
\hline $\begin{array}{c}\text { ENTRENO } \\
\text { PRE }\end{array}$ & 4 & 5 & 9 & 4 \\
\hline
\end{tabular}

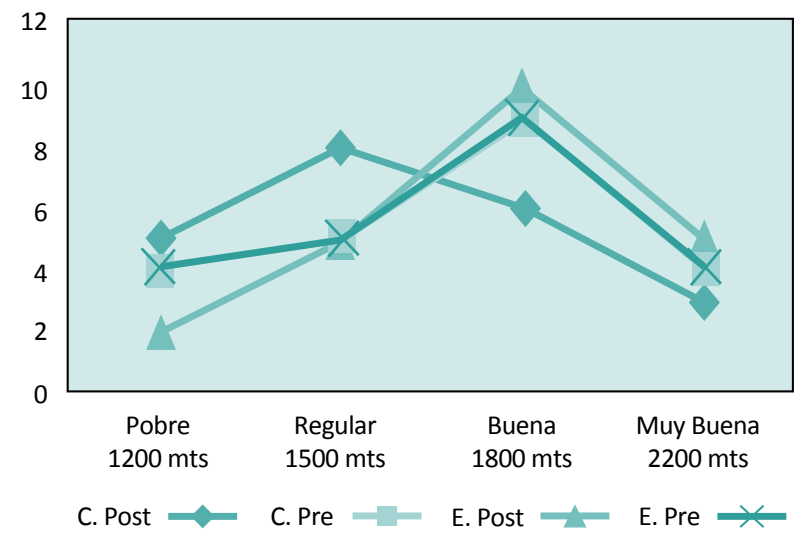

Figura 3.

No se observó efecto significativo del grupo ni del tiempo ( $p>0,05)$, y se observó un efecto significativo de la interacción grupo por tiempo 
$(\mathrm{p}=0,018)$ para la prueba de fuerza de presión de las piernas 1RM (Figura 4). En el grupo de intervención la fuerza de presión de las piernas aumentó significativamente $(+32 \%)$ con el entrenamiento. La fuerza muscular de las piernas tendió a disminuir durante el periodo de estudio en el grupo control, aunque no se alcanzó la significación estadística en las comparaciones Posthoc dentro de este grupo $(\mathrm{p}=>0,05)$.

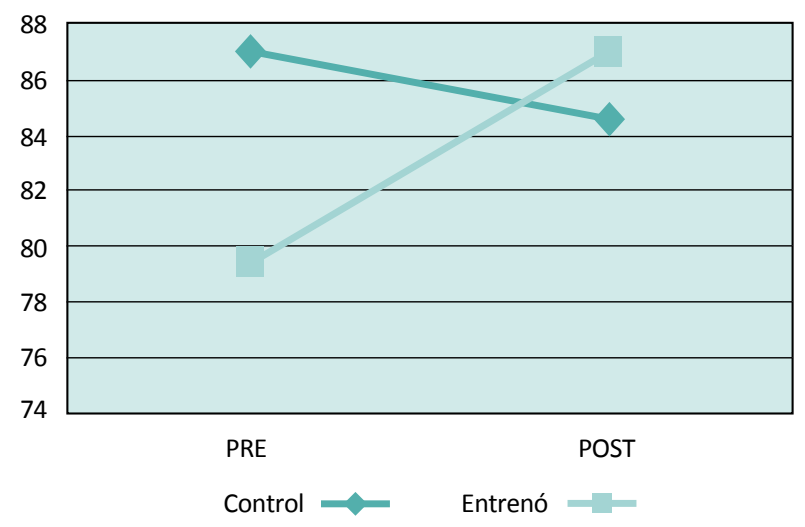

Figura 4. Fuerza de piernas (press banc).

No se observó efecto significativo del grupo ni del tiempo ( $p>0,05)$, y se observó un efecto significativo de la interacción grupo por tiempo $(\mathrm{p}=0,018)$ para la prueba de fuerza de presión de los brazos 1RM (Figura 5). En el grupo de intervención la fuerza de presión de los brazos aumentó significativamente $(+18,26 \%)$ con el entrenamiento. La fuerza muscular de los brazos tendió a aumentar durante el periodo de estudio en el grupo control pero en solo un 4,34\%, aunque no se alcanzó la significación estadística en las comparaciones Post-hoc dentro de este grupo $(\mathrm{p}=>0,05)$.

\section{Tabla 5. Fuerza de brazos (press banc)}

\begin{tabular}{ccc}
\hline VARIABLES & PRE PROGRAMA & $\begin{array}{c}\text { POST } \\
\text { PROGRAMA }\end{array}$ \\
\hline Peso kg control & 57,5 & 60 \\
\hline Peso kg entreno & 57,5 & 68 \\
\hline
\end{tabular}

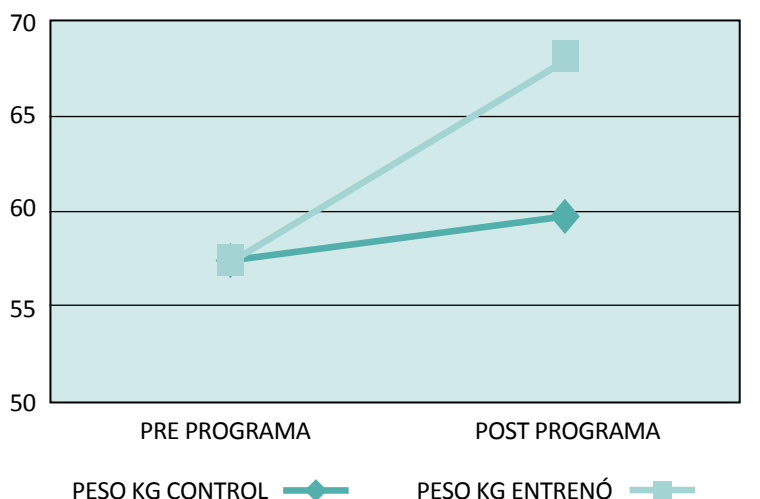

Figura 5.

\section{Resultados secundarios}

No se observó efecto significativo del grupo ni del tiempo ( $p>0,05)$, y se observó un efecto significativo de la interacción grupo por tiempo $(\mathrm{p}=0,018)$ para la prueba de fuerza resistencia de las piernas (Figura 6). En el grupo de intervención la fuerza resistencia de las piernas aumentó significativamente $(+15,2 \%)$ con el entrenamiento. La fuerza resistencia de las piernas muscular se mantuvo igual durante el periodo de estudio en el grupo control, no se alcanzó la significación estadística en las comparaciones Posthoc dentro de este grupo $(p=>0,05)$.

\section{Tabla 6. Fuerza resistencia de piernas (escalón de Harvard)}

\begin{tabular}{ccc} 
VARIABLES & EVA PRE & EVA POST \\
\hline ENTRENO & 40 & 44 \\
CONTROL & 40 & 40 \\
\hline
\end{tabular}

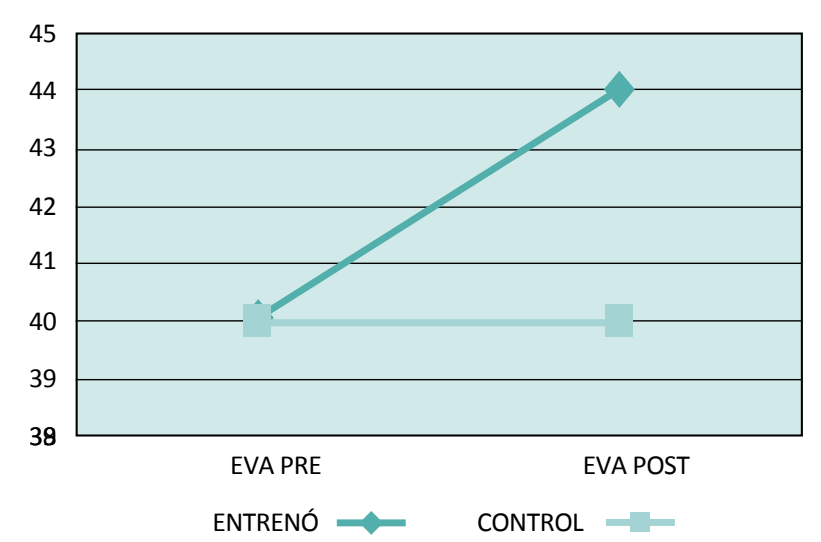

Figura 6. 
No se observó efecto significativo del grupo ni del tiempo ( $p>0,05)$, y se observó un efecto significativo de la interacción grupo por tiempo $(\mathrm{p}=0,018)$ para la prueba de fuerza resistencia de los brazos (Figura 7). En el grupo de intervención la fuerza resistencia de los brazos aumentó significativamente $(+33,33 \%)$ con el entrenamiento. La fuerza resistencia de los brazos se mantuvo igual durante el periodo de estudio en el grupo control, no se alcanzó la significación estadística en las comparaciones Post-hoc dentro de este grupo $(p=>0,05)$.

Tabla 7. Fuerza resistencia de brazos (dominadas)

\begin{tabular}{ccc}
\hline VARIABLES & EVA PRE & EVA POST \\
\hline ENTRENO & 1,5 & 2 \\
CONTROL & 1,5 & 1,5 \\
\hline
\end{tabular}

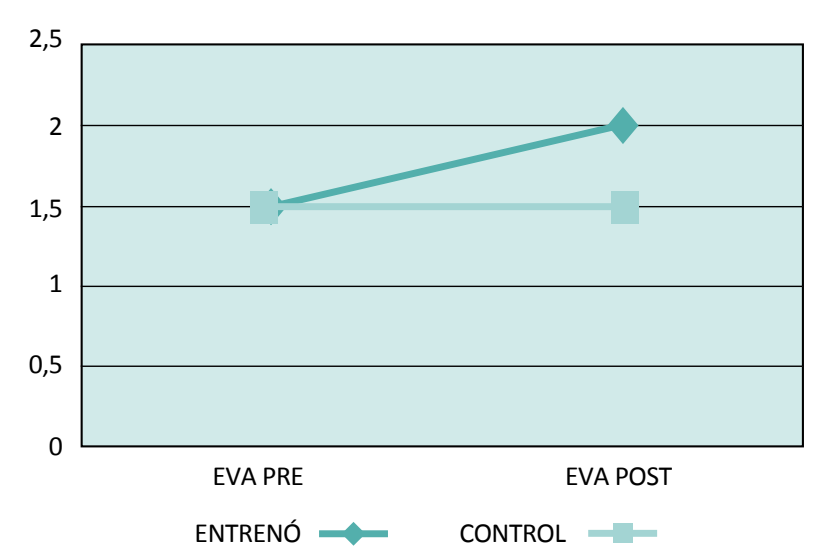

Figura 7.

No se observó efecto significativo del grupo ni del tiempo ( $p>0,05)$, y se observó un efecto significativo de la interacción grupo por tiempo $(\mathrm{p}=0,018)$ para la prueba del peso y el índice de masa corporal (Figura 8 y 9 respectivamente). En el grupo de intervención disminuyó en peso en $+8,72 \%$ y el índice de masa corporal disminuyó en un $9,7 \%$ con el entrenamiento. El peso en el grupo control disminuyó solo en un $2,7 \%$ y el IMC disminuyó un 4\%, no se alcanzó la significación estadística en las comparaciones Post-hoc dentro de este grupo $(p=>0,05)$.
Tabla 8. Peso corporal

\begin{tabular}{ccc} 
VARIABLES & $\begin{array}{c}\text { PRE } \\
\text { PROGRAMA }\end{array}$ & $\begin{array}{c}\text { POST } \\
\text { PROGRAMA }\end{array}$ \\
PESO KG & 86,87 & 84,5 \\
CONTROL & & 79,29 \\
PESO KG & 86,87 & 7 \\
\hline
\end{tabular}

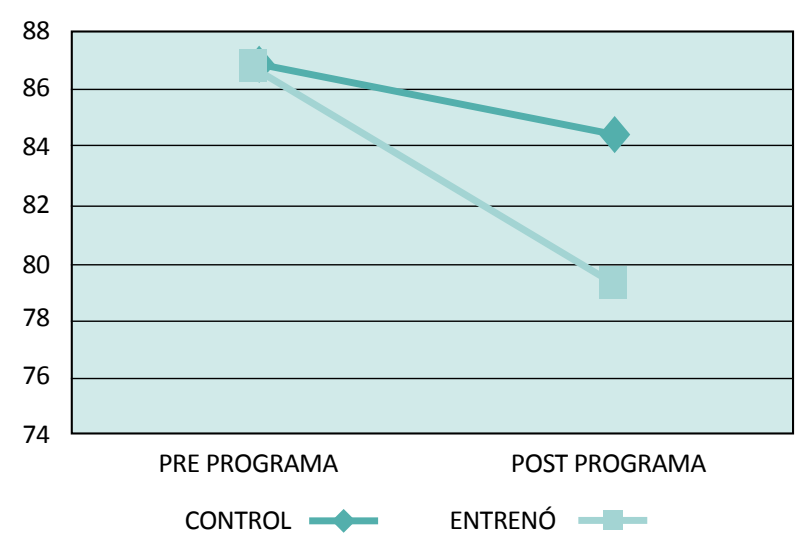

Figura 8

Tabla 9. Índice de masa corporal

\begin{tabular}{ccc} 
VARIABLES & $\begin{array}{c}\text { PRE } \\
\text { PROGRAMA }\end{array}$ & $\begin{array}{c}\text { POST } \\
\text { PROGRAMA }\end{array}$ \\
\hline IMC Entreno & 31,25 & 28,23 \\
IMC Control & 31,25 & 30 \\
\hline
\end{tabular}

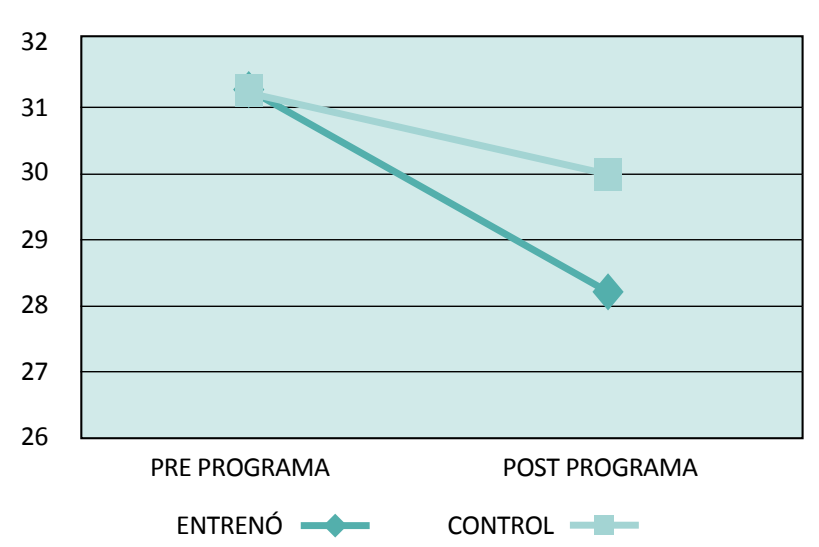

Figura 9.

No se observó efecto significativo del grupo ni del tiempo ( $p>0,05)$, y se observó un efecto significativo de la interacción grupo por tiempo $(\mathrm{p}=0,018)$ para la prueba la FCM Y FCR (Fi- 
gura 10). En el grupo de intervención disminuyó la FCM en un 7,7 \% con el entrenamiento y la FCR disminuyó un 9,47 \%, el grupo control disminuyó hasta en $1,8 \%$ en la FCM y $2,1 \%$ en la FCR. No se alcanzó la significación estadística en las comparaciones Post-hoc dentro de este grupo $(p=>0,05)$.

\section{Tabla 10. Valoración de la frecuencia cardiaca máxima y en reposo}

\begin{tabular}{cccc}
\multicolumn{2}{c}{ VARIABLES } & Preprograma & Postprograma \\
G. Entreno & FCM & 168 & 155 \\
& FCR & 95 & 90 \\
\multirow{2}{*}{ G. Control } & FCM & 168 & 165 \\
& FCR & 95 & 93 \\
\hline
\end{tabular}

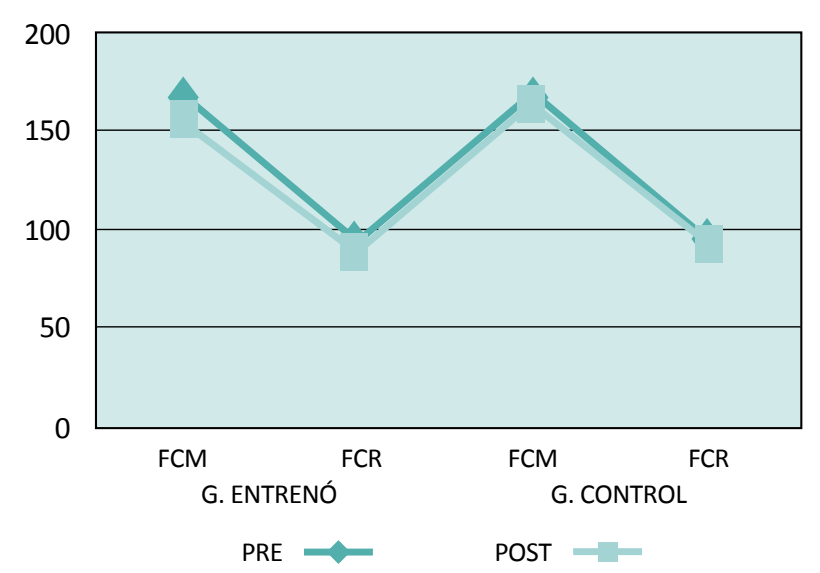

Figura 10.

\section{CONCLUSIONES}

Se concluye que los efectos que un programa de la fuerza muscular de doce semanas de duración, genera mejora en la condición física de los participantes, así como en la fuerza muscular y en la fuerza resistencia, además de generar ganancias en la velocidad de la marcha, disminuye el peso corporal y el IMC, así como también la FCM y FCR. Las recomendaciones del presente estudio es continuar con este tipo de estudios y con un número mayor de participantes.

\section{REFERENCIAS}

1. Gottlob. A, (2008).Entrenamiento muscular diferenciado. Editorial Paidotribo, Les Guixeres, C/ de la Energía, 19-21 08915. Barcelona, España.

2. Salazar P. D, J. Ramírez V. D. Chaparro, (2014). Revisión sistemática sobre el impacto de la actividad fisica en los trastornos de la marcha en el adulto mayor. Disponible en: http://dx.doi.org/10.5672/ apunts.2014-0983.es.(2014/4).118.03.

3. Jacoby, E. Bull, F. \& Neiman, A. (2003). Rapid changes in lifestyle make increased physical activity a priority for the Americas. Revista Panamericana de Salud Pública, 14(4):

http://www.scielosp.org/scielo.php?script=sci_artte

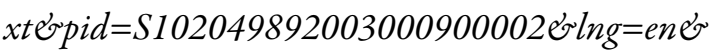
nrm=iso $\mathrm{e}$ tlng $=$ en

4. González Peris (2009). Prescripción del ejercicio fisico, la mejor receta para la salud:

http://historico.medicosypacientes.com/noticias/2009/02/09_02_14_ejercicio

5. Mendieta. L, Yaguachi. F, Toledo. K. (2013). Programa de entrenamiento para el incremento de la fuerza muscular. Disponible en:

http://www.uap.edu.pe/Investigaciones/Esp/Revista_16-02_Esp_05.pdf

6. García, Gil, M. (2012). Manual de ejercicio físico para personas de edad avanzada. Disponible en: http://www.bizkaia.net/home2/archivos/DPTO4/Temas/manual-cast-ultima. $p d f$ ? idioma $=C A$

7. Lusa Cadore. E, Silveira Pinto. R, Bottaro. M, Izquierdo. M. (2014). Strength and Endurance Training Prescription in Healthy and Frail Elderly. Published online 2014 Jun 1. Prepublished online 2013 Oct 24. doi: 10.14336/AD.2014.0500183 PMCID: PMC4037310 
8. Tiffany N. Frimel, David R. Sinacore, Dennis T. Villareal. (2009). Exercise Attenuates the Weight-Loss-Induced Reduction in Muscle Mass in Frail Obese Older Adults. Med Sci Sports Exerc. Author manuscript; available in PMC 2009 Mar 3. Published in final edited form as: Med Sci
Sports Exerc. 2008 Jul; 40(7): 1213-1219. doi: 10.1249/MSS.0b013e31816a85ce PMCID: PMC2650077 NIHMSID: NIHMS92276.

http://www.ncbi.nlm.nih.gov/pmc/articles/ PMC2650077/ 


\section{ANEXOS}

\section{Anexo 1. Consentimiento informado por escrito}

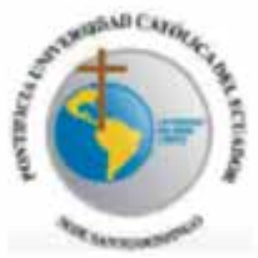

\section{PONTIFICIA UNIVERSIDAD CATÓLICA DEL ECUADOR}

Entrenamiento de la fuerza muscular más la función cardiovascular para

mejoramiento de la salud en adultos de Santo Domingo

\section{CONSENTIMIENTO INFORMADO POR ESCRITO}

EANTO DOMINGO, a de de 20

Yo. declaro ilore y voluntariamente que acepte participar en las sesiones de Entrenamiento de la fuerza muscular mas la suncion cardovascular que se realzaran en el Derviclo de Entrenamiento Medico Depertivo en las areas de fisiologia del ejercicio fiako, cujos objetivos conaleten en:

- Mejorartherza musculgr

- Mejorar el rendimiento aersoico y mejorar ia capacidad anaerboica

- Mejorar ia salud

- Combarar las gananclas con etro gupe etano del mismo centro

Estoy consciente de que los procedimientos, pruebas y tratamientos para lograr los cbjetivos mencionados consiatran en:

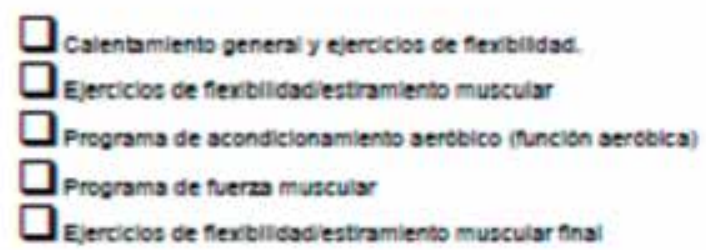

Los resgos en caso de que existan, en m persona inherente a las pruebss que se resizaran, me fueron exilcados verbaimente, los entendi en su totaldad y los actpto de conformidad.

Es de mi conocimiento que sere itere de retirarme del programa de ejercicio en ei momento que yo asl io desee. Tambien que puedo solicitar informacion adcicns acerca de los nespos y beneficios de mi participacion en el programa. En caso de que cecldera retrarme, la atencion que como paciente recibo en esta instivicion no se vera afectads.

Nombre.

Fima.

Direocton Fecha.

Fima de padre e tuter (en case de cer mener de odad)

Tolatence

Toctigo Firma. Dirtooblon 


\section{Anexo 2. Diagrama de flujo}

$\mathrm{N}=58$ sexagenarios de Santo Domingo

$\mathrm{N}=48$ superaron los arterios de idoneidad

Motivos: enfermedad aguda 5; insuficiencia cardíaca 6 , fractura de las extremidades inferiores 4; no permitió el consentimiento 9 .

Grupo entrenó $\mathrm{N}=22$ 19 mujeres y 3 hombres; programa de entrenamiento para la fuerza muscular, cinco días a la semana, 12 semanas

22 evaluados

\section{$\mathrm{N}=44$ Aleatorizado}

Inicio de intervención

$\mathrm{N}=465$ sesiones de familiarización y medidas previas a la intervención

2 decidieron no participar

Grupo control N= 2 hombres y 20 mujeres
Después de la intervención
22 evaluados 


\title{
Anexo 3. Certificados
}

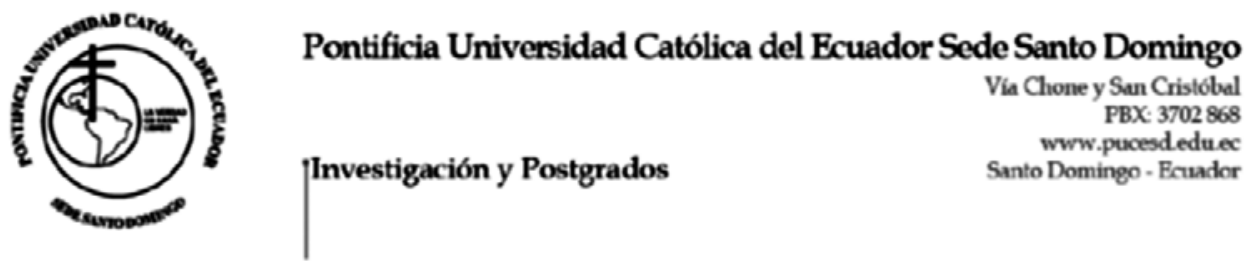

\section{CERTIFICADO MÉDICO PARA INVESTIGACIÓN}

\author{
El suscrito médico general. Alicia Fernanda Yaguachi Orellana, con COD MSP \\ 37 FOLIO 58 N175.
}

\section{Certifica}

Que habiendo practicado un reconocimiento médico el dia jueves veinte y siete de noviembre de dos mil catorce (27/11/2014) al paciente denominado número uno (1), lo encontré: en buen estado de salud fisica sin defectos ni anomalias en el aparato locomotor; presenta una integra capacidad cognitiva, agudeza visual y auditiva; aparatos cardiovascular y respiratorio en óptimas condiciones; el examen clínico y antropométrico revelaron altos indices de LDL, colesterol general, glicemia y tensión arterial, así como sobrepeso.

Por lo anterior se hace constar que el paciente, puede participar del programa de actividad física recomendado por el maestro Lenin Mendieta Toledo.

El presente certificado médico es expedido a petición del programa de investigación para establecer los efectos del mismo sobre el estado de salud de los participantes

Es cuanto puedo certificar en honor a la verdad y autorizo a los investigadores hacer uso del presente para los fines pertinentes.

Atentamente,

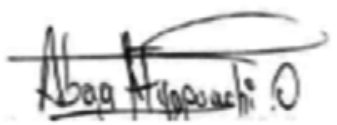

Dra. Alicia Fernanda Yaguachi Orellana COD MSP 37 FOLIO 58 N175.

Santo Domingo, 27 de marzo de 2015 


\section{Anexo 4. Certificados}

\section{CERTIFICADO PARA INVESTIGACIÓN}

Dra. Alicia Fernanda Yaguachi Orellana, con COD MSP 37 FOLIO 58 N175. Certifico haber monitoreado el estudio "Entrenamiento de la fuerza muscular más la función cardiovascular para mejoramiento de la salud en adultos mayores del Hospital de Santo Domingo", que se realizó en la ciudad de Santo Domingo de los Tsáchilas, con pacientes adultos mayores, desde el dia uno de diciembre de dos mil catorce (1/12/14) hasta el dia veinte y siete de marzo de dos mil quince (27/3/15).

Es cuanto puedo certificar en honor a la verdad y autorizo a los investigadores hacer uso del presente para los fines pertinentes.

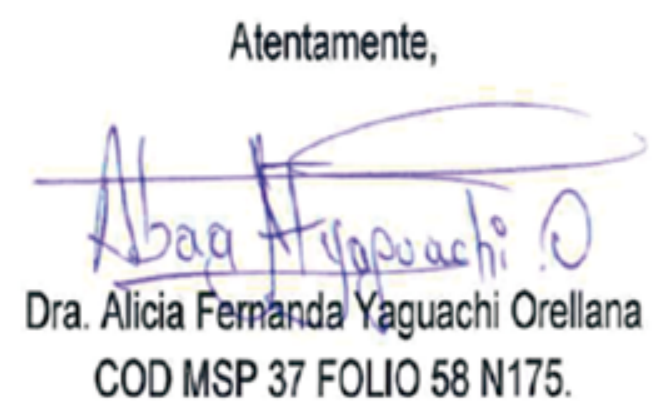

Santo Domingo, 27 de marzo de 2015 


\section{Anexo 5. Prescripción médica del ejercicio}

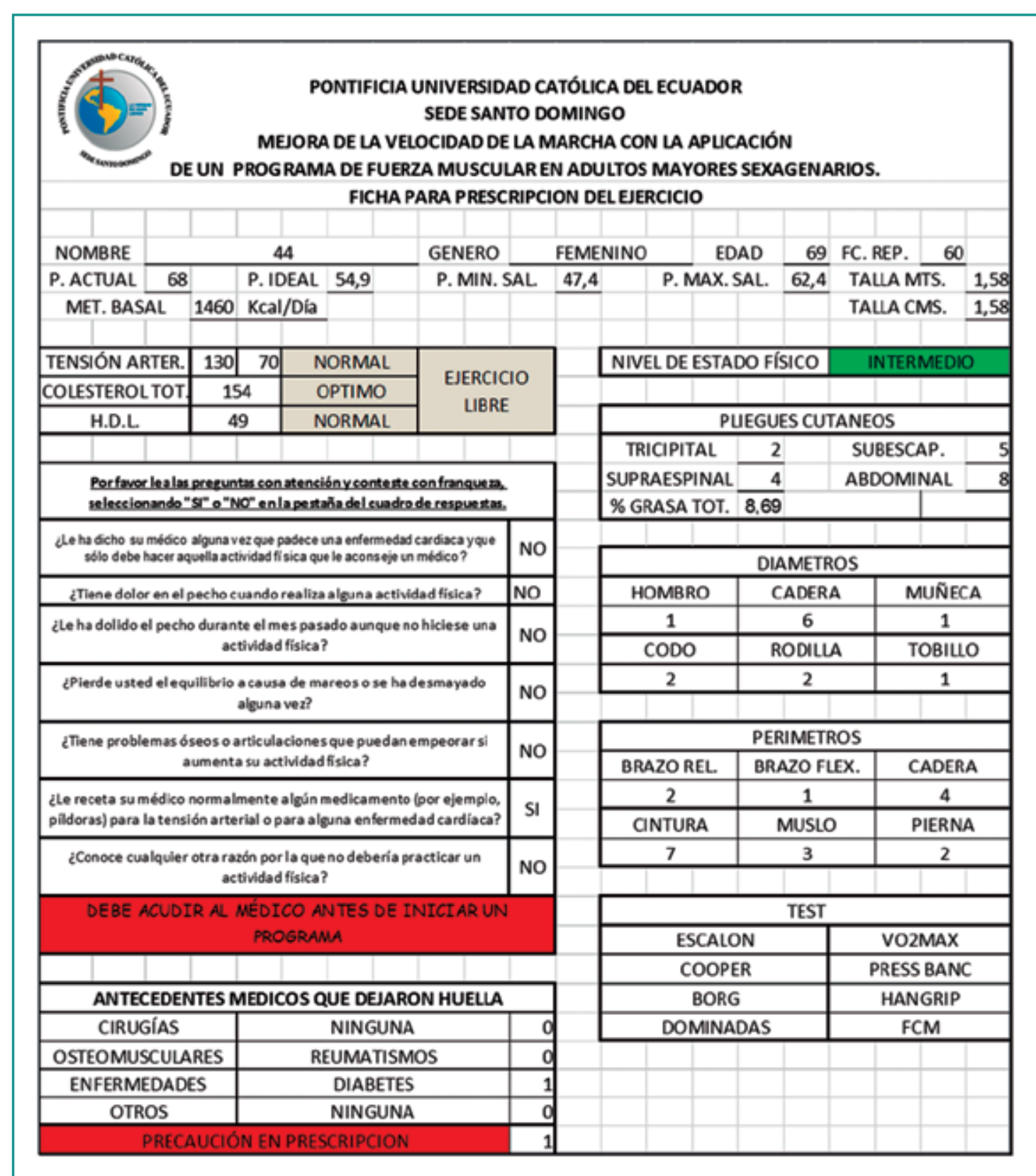

